\title{
机械伤害和外源茉莉酸诱导踠豆幼苗 $\mathrm{H}_{2} \mathrm{O}_{2}$ 系统性产生 ${ }^{*}$
}

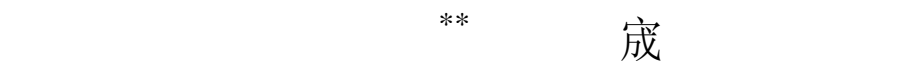

(1) 中国农业大学食品科学与营养工程学院, 北京 100083; (2) 内蒙古农业大学农学院, 呼和浩特 100018)

摘要 以踠豆(Pisum sativum L.)幼苗为试材, 采用 DAB 组织染色、 $\mathrm{CeCl}_{3}$ 细胞化学染色和 $\mathrm{TiCl}_{4}$ 显色等多种方法, 跟踪了伤害和外源茉莉酸(jasmonic acid, JA)处理后 $\mathrm{H}_{2} \mathrm{O}_{2}$ 的时空动态变化. 结果 显示, 伤害和外施 JA 可以诱导 $\mathrm{H}_{2} \mathrm{O}_{2}$ 系统性产生, 无论是伤害叶片、邻近未伤害叶片还是低节位 远端叶片, 伤害处理后 $1 \mathrm{~h}, \mathrm{H}_{2} \mathrm{O}_{2}$ 含量即有所增加, 3 5 h 后 $\mathrm{H}_{2} \mathrm{O}_{2}$ 含量达到最大, 随后开始下降, 至 处理后 $12 \mathrm{~h}, \mathrm{H}_{2} \mathrm{O}_{2}$ 含量基本恢复到对照水平; 相应地, 伤害和 JA 处理后, 抗氧化酶类活性迅速提 高; NADPH 氧化酶抑制剂二苯基碘(diphenylene iodonium, DPI)可以显著抑制伤害和 JA 诱导的 $\mathrm{H}_{2} \mathrm{O}_{2}$ 迸发. $\mathrm{H}_{2} \mathrm{O}_{2}$ 亚细胞定位结果显示, 伤害和 JA 诱导产生的 $\mathrm{H}_{2} \mathrm{O}_{2}$ 主要分布于质膜、细胞壁和 细胞间隙. JA 的胶体金免疫电子显微镜定位结果表明, 伤害后 JA 含量迅速增加, 显示在叶肉细 胞的细胞壁和韧皮部簰管与伴胞分子中金颗粒数量明显增加.
\end{abstract}

关键词伤害 茉莉酸 $\mathrm{H}_{2} \mathrm{O}_{2}$ 组织化学定位 细胞化学定位 胶体金免疫电子显微镜定位

机械胁迫是植物经常遭受的逆境之一, 当前食 品行业中果蔬净加工的兴起和深加工的快速发展, 还出现了人为造成的伤害. 同遭受其他逆境一样, 植 物对机械伤害也有其适应性机制 $[1]$. 茉莉酸及其甲酯 (methyl jasmonate, MeJA) 被认为是植物伤信号分子, 其在植物防御反应中的作用得到了人们的广泛关注. 在马铃薯 ${ }^{[2]}$ 、烟草 ${ }^{[3]}$ 、番茄 ${ }^{[4]}$ 等作物上的研究表明, 内 外源JA/MeJA均对植物伤害防卫基因的表达具有调
节和促进作用. 但目前, 人们对JA提高植物抗逆性机 制的了解尚不足.

氧化突发(oxidative burst, OXB), 即植物细胞在 胁迫条件下迅速产生一些活性氧分子(reactive oxygen species, ROS), 被认为是植物具有的一种先天的 “免 疫系统” [5 7]. 以往对于氧化突发机制的研究多是建立 在植物受病原菌侵染的模式下, 近十多年来研究发现, 多种非生物因素, 如渗透胁迫 ${ }^{[8]}$ 、UV辐射 ${ }^{[9]}$ 等也可以 
诱导植物的OXB. 研究还发现, 伤害胁迫也会诱导植 物组织产生并累积ROS ${ }^{[4,10]}$. 由于活性氧的寿命十分 短暂, 反应活性特别强, 为活性氧检测带来很大难度, 同时, 由于以往的研究多采用悬浮细胞系, 无法检测 系统诱导作用, 因此, 有关活性氧在植物局部获得性 抗性(local acquired resistance, LAR)或系统获得性抗 性(systemic acquired resistance, SAR)中的作用仍存在 很多争论. $\mathrm{H}_{2} \mathrm{O}_{2}$ 被认为是植物体内产生的最稳定的活 性氧分子, 本试验以整株踠豆幼苗为试材, 分别采用 组织化学原位检测、细胞化学定位和 $\mathrm{TiCl}_{4}$ 显色等多种 方法, 跟踪了伤害和JA处理后 $\mathrm{H}_{2} \mathrm{O}_{2}$ 产生的时间和空 间动态变化，同时，利用胶体金免疫电子显微镜技术 对伤害后叶片中JA的分布和数量进行了亚细胞定位, 旨在了解 $\mathrm{H}_{2} \mathrm{O}_{2}$ 在伤害防御反应中的作用, 并为进一 步揭示JA作为伤信号分子的作用机制提供依据.

\section{1 材料与方法}

\section{1 植物材料}

选生长健壮, 无病虫害的 10 日龄已长有 6 片叶 子的宁夏豌豆(Pisum sativum L.)幼苗为试材, 材料由 中国农业大学马连洼温室提供. 将材料从茎基部切 下置于盛水的白瓷盘中, 置于 $25^{\circ} \mathrm{C}$, 光强 $200 \mu \mathrm{mol} /$ $\left(\mathrm{m}^{2} \cdot \mathrm{s}\right)$ 的光照培养箱中, 缓苗 $24 \mathrm{~h}$ 后开始处理.

\section{2 材料处理}

(1) 伤害处理: 用医用剪刀迅速将踠豆幼苗上部 一片完全展开叶剪伤, 伤及主脉, 在伤害处理后 0,1 , $3,5,8,12,24 \mathrm{~h}$, 分别取伤害叶片、相邻未伤害叶片以 及同株低节位未伤害叶片, 一部分立即固定, 用于 $\mathrm{H}_{2} \mathrm{O}_{2}$ 和 JA 亚细胞定位; 一部分液氮速冻, 用于 $\mathrm{H}_{2} \mathrm{O}_{2}$ 含量及抗氧化酶类活性分析. 未经伤害处理的材料 作为对照, 与伤害处理同期取样, 每个处理重复 3 次.

(2) 药剂处理: 将材料茎基部置于含 $10 \mu \mathrm{mol} /$ $\mathrm{L}( \pm)-\mathrm{JA}$ (购自 Sigma 公司)溶液中, 并在 $25^{\circ} \mathrm{C}$, 光强 $200 \mu \mathrm{mol} /\left(\mathrm{m}^{2} \cdot \mathrm{s}\right)$ 的光照培养箱中放置 $0,1,3,5,8,12$, $24 \mathrm{~h}$, 取植株中部完全展开叶, 一部分用于 $\mathrm{H}_{2} \mathrm{O}_{2}$ 亚细 胞定位, 一部分用于 $\mathrm{H}_{2} \mathrm{O}_{2}$ 含量及抗氧化酶类活性分 析. 对照为水处理.

DPI(购自 Sigma 公司)是 NADPH 氧化酶自杀性
底物抑制剂, 同样采用茎基部施入法, 处理浓度为 $100 \mu \mathrm{mol} / \mathrm{L}$, 置于 $25^{\circ} \mathrm{C}$, 光强 $200 \mu \mathrm{mol} /\left(\mathrm{m}^{2} \cdot \mathrm{s}\right)$ 的光 照培养箱中吸收 $4 \mathrm{~h}$, 之后一部分转入水中并对材料 进行伤害处理, 一部分转入含 $10 \mu \mathrm{mol} / \mathrm{L}( \pm)-\mathrm{JA}$ 的溶 液中, 分别在处理后 $0,1,3,5,8,12,24 \mathrm{~h}$ 取样, 方法 同上. 对照为水处理.

\section{$1.3 \mathrm{H}_{2} \mathrm{O}_{2}$ 活体组织化学原位检测}

参照 Thordarl-Christense 等 人 [11] 和 OrozcoCardenas等人 ${ }^{[12]}$ 的方法, 将豌豆幼苗茎基部浸泡在 1 $\mathrm{mg} / \mathrm{mL}$ 的 $\mathrm{DAB}$ 溶液中, 置于 $25^{\circ} \mathrm{C}$, 光强 200 $\mu \mathrm{mol} /\left(\mathrm{m}^{2} \cdot \mathrm{s}\right)$ 的光照培养箱中照光 $4 \mathrm{~h}$, 之后将材料 一部分进行伤害处理, 一部分转入含 $10 \mu \mathrm{mol} / \mathrm{L} \mathrm{JA}$ 的 DAB溶液中处理，分别在处理后 $0,1,3,5,8,12,24 \mathrm{~h}$ 取样，立即投入到 96\%的乙醇中煮沸 $10 \mathrm{~min}$ 脱色，然 后在新鲜的乙醇中浸泡 $4 \mathrm{~h}$, 目测叶片中红褐色沉淀 量并照相.

\section{$1.4 \mathrm{H}_{2} \mathrm{O}_{2}$ 细胞化学定位}

参照Bestwick等人 ${ }^{[13]}$ 和 Pellinen等人 ${ }^{[14]}$ 的方法, 通过 $\mathrm{CeCl}_{3}$ 沉淀对 $\mathrm{H}_{2} \mathrm{O}_{2}$ 进行亚细胞定位. 分别取经过 伤害处理和药剂处理的叶片, 将材料分割成(1 2) $\mathrm{mm} \times(3 \sim 5) \mathrm{mm}$ 大小, 迅速投入到 $50 \mathrm{mmol} / \mathrm{L} \mathrm{MOPS}$ 缓 冲液中 $\left(\mathrm{pH}\right.$ 7.2), 内含 $5 \mathrm{mmol} / \mathrm{L} \mathrm{CeCl}_{3}$, 以不含 $\mathrm{CeCl}_{3}$ 的MOPS溶液为空白对照, 真空抽气 $0.5 \mathrm{~h}$, 用 50 $\mathrm{mmol} / \mathrm{L}$ 二甲砷酸钠缓冲液( $\mathrm{CAB}, \mathrm{pH} 7.2$ )含 $1.25 \%$ 戊 二醛和 $1.25 \%$ 多聚甲醛，室温下固定 $1 \mathrm{~h}, \mathrm{CAB}$ 充分洗 涤, 然后在含 $1 \%$ 锇酸的 $\mathrm{CAB}$ 溶液中后固定 $8 \mathrm{~h}$, 经 $\mathrm{CAB}$ 充分洗涤后，不同浓度酒精系列脱水，丙䣶过渡， Spure包埋, $60^{\circ} \mathrm{C}$ 高温聚合 48 h, LKB-8800 型切片机 超薄切片, 切片经铀-铅双染后在JEM-100 电子显微 镜下观察并照相.

\section{$1.5 \mathrm{H}_{2} \mathrm{O}_{2}$ 含量测定}

参照Patterson等人近]的方法, 以标准 $\mathrm{H}_{2} \mathrm{O}_{2}$ 液做 标准曲线, 结果用 $\mathrm{H}_{2} \mathrm{O}_{2} \mathrm{nmol} / \mathrm{g} \mathrm{FW}$ 表示.

\section{6 超氧化物歧化酶(SOD)、过氧化氢酶(CAT)、 超氧阴离子自由基 $\left(\mathrm{O}_{2}^{--}\right)$产生速率的测定}

分别取 $0.5 \mathrm{~g}$ 叶片, 加入 $5 \mathrm{~mL}$ 提取缓冲液(50 
$\mathrm{mmol} / \mathrm{L}$ 磷酸缓冲液, $1 \% \mathrm{PVPP}, 5 \mathrm{mmol} / \mathrm{L} \mathrm{DTT}, \mathrm{pH}$ $7.8)$, 冰浴研磨成浆, $12000 \times g, 4^{\circ} \mathrm{C}$ 下离心 $20 \mathrm{~min}$, 上 清液为酶提取液.

SOD测定参照Dhindsa等人 $[16]$ 的方法，以抑制 NBT光化学还原 $50 \%$ 为一个酶活力单位, 结果用 $\mathrm{U} /(\mathrm{mg}$ Pr ・ min) 表示. CAT活性测定参照EI-Moshaty等 人 ${ }^{[17]}$ 的方法, 结果用 $1 \mathrm{~min}$ 内 $A_{240}$ 减少 0.1 为一个酶活 力单位表示. $\mathrm{O}_{2}^{-*}$ 产生速率测定参照王爱国等人 ${ }^{[18]}$ 和 苏新国等人 ${ }^{[19]}$ 的方法, 以 $\mathrm{NO}_{2}^{-}$作标准曲线, 结果用 $\mathrm{nmol} \mathrm{NO}-/(\operatorname{mgPr} ・ \min )$ 表示.

\section{7 抗坏血酸过氧化酶(APX)活性测定}

参照沈文遂等人 ${ }^{[20]}$ 的方法, 以加入 $\mathrm{H}_{2} \mathrm{O}_{2}$ 为反应 开始, 测定 $30 \mathrm{~s}$ 内 $A_{290}$ 的变化值, 结果用 $\Delta A_{290} /(\mathrm{mg}$ Pr・min)表示.

\subsection{JA 胶体金免疫电子显微镜定位}

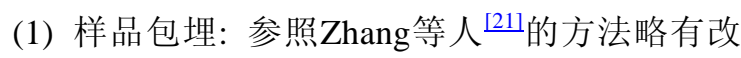
动. 分别取伤害和对照的叶片, 将材料分割成(1 2) $\mathrm{mm} \times(3 \sim 5) \mathrm{mm}$ 大小并迅速投入 $2 \% \operatorname{EDC}(w / v)$ 溶液中, 抽气 $0.5 \mathrm{~h}$ 至材料下沉, 之后转入含 $4 \%$ 多聚甲醛和 $1 \%$ 戊二醛的磷酸缓冲液 $(\mathrm{pH} 7.2)$ 中 $4^{\circ} \mathrm{C}$ 下固定 $8 \mathrm{~h}$, 经 磷酸缓冲液充分洗涤后, 酒精系列脱水, $100 \%$ 酒精LR-white置换及纯 LR-white浸透过夜, 最后包埋于 $0.5 \mathrm{~mL}$ 离心管中, $48^{\circ} \mathrm{C}$ 聚合 $48 \mathrm{~h}, \mathrm{LKB}-8800$ 型切片机 超薄切片, 切片捞在 150 目以 $0.3 \%$ Formar膜覆盖的 镍网上.

（2）免疫染色: 参照王永章等人 ${ }^{[22]}$ 的方法略有改 动. 超薄切片用 TBSTG (1: 50 正常牛血清/TBST (0.15 mol/L Tris-HCl pH 7.6, $0.15 \mathrm{~mol} / \mathrm{L} \mathrm{NaCl,} 1 \%$ Toween 20,1\%明胶))阻断 $30 \mathrm{~min}$ 以除去非特异结合 位点, 切片不经清洗, 直接在 1: 100 JA 单克隆抗体 /TBSTG 溶液中 $4{ }^{\circ} \mathrm{C}$ 下孵育过夜, TBSTG 充分洗涤 (4×5 min), 然后在 $1: 40$ 胶体金标记(12 nm)的羊抗 鼠抗体/TBSTG 溶液中室温下孵育 $1 \mathrm{~h}$, 依次经 TBSTG, TBST, $\mathrm{ddH}_{2} \mathrm{O}$ 充分洗涤, 切片再经铀-铅双染 后在 JEM-100 电子显微镜下观察并照相.

为了检验免疫定位方法的可行性和试验结果的 可靠性, 分别设立了 2 种负对照: 以免疫前的小鼠血
清代替 JA 单克隆抗体, 忽略一抗以检验与二抗结合 的特异性. 免疫程序的其他步骤同正常免疫染色. 每 个样品处理重复 3 5 次.

\section{2 结果与分析}

\section{1 机械伤害和 $\mathrm{JA}$ 诱导影豆叶片 $\mathrm{H}_{2} \mathrm{O}_{2}$ 产生的组 织化学定位}

在有过氧化物酶存在下, $\mathrm{DAB}$ 一旦与 $\mathrm{H}_{2} \mathrm{O}_{2}$ 接触 就会迅速形成红褐色聚合物沉淀, 因此, 可以通过直 接目测颜色深浅及其出现的部位来分析 $\mathrm{H}_{2} \mathrm{O}_{2}$ 的数量 和存在部位. 如图 1(a)所示, 未伤害的对照叶片, 红 褐色 DAB- $\mathrm{H}_{2} \mathrm{O}_{2}$ 沉淀很少, 整个处理过程中叶片颜色 几乎没有变化. 伤害叶片在伤害后 $1 \mathrm{~h}, \mathrm{H}_{2} \mathrm{O}_{2}$ 含量即 有所增加, 且随着处理时间延长, 叶片颜色逐渐加深; 其中, 伤害后 1 3 h 叶片颜色加深辐度最大, 伤害后 3 12 h 叶片颜色加深辐度有所减弱, 推测伤害后 1 3 $h$ 可能是 $\mathrm{H}_{2} \mathrm{O}_{2}$ 产生的高峰期. 图 1(b)进一步表明, $\mathrm{H}_{2} \mathrm{O}_{2}$ 最早产生于伤害位点, 随后扩展到叶片的主脉、 细脉以及叶肉细胞中, 在相对应的邻近未伤害叶片 中也有大量 DAB- $\mathrm{H}_{2} \mathrm{O}_{2}$ 沉淀出现.

外施 $10 \mu \mathrm{mol} / \mathrm{L} \mathrm{JA}$ 后, 叶片红褐色出现的面积增 加, 颜色加深, 表明 JA 可以诱导 $\mathrm{H}_{2} \mathrm{O}_{2}$ 产生, 且随着 JA 处理时间延长, DAB- $\mathrm{H}_{2} \mathrm{O}_{2}$ 沉淀明显增加(图 1(c)).

DPI 是 NADPH 氧化酶特异性抑制剂, 与直接进 行伤害处理的叶片相比, DPI 处理后 $4 \mathrm{~h}$ 再进行伤害 处理, 叶片中红褐色沉淀明显减少(图 1(d)). 同样, 经 DPI 处理的材料外源施加 JA 后, 红褐色沉淀也明 显减少(资料未显示).

\section{2 机械伤害和 $\mathrm{JA}$ 诱导影豆叶片 $\mathrm{H}_{2} \mathrm{O}_{2}$ 产生的细 胞化学定位}

在电子显微镜下明显观察到，未经伤害处理的 叶片, 叶肉细胞中 $\mathrm{H}_{2} \mathrm{O}_{2}-\mathrm{CeCl}_{3}$ 沉淀颗粒很少, 只在质 膜和叶绿体基粒片层上有少量分布(图 2(a), (b)). 当 孵育液中不加底物 $\mathrm{CeCl}_{3}$ 时, 未能观察到黑色颗粒状 沉淀(图 2(c)), 因此, 本试验中, $\mathrm{CeCl}_{3}$ 沉淀颗粒多少 以及着色深浅为 $\mathrm{H}_{2} \mathrm{O}_{2}$ 含量的真实反映.

与未经伤害处理的对照相比, 伤害叶片叶肉细胞 中 $\mathrm{H}_{2} \mathrm{O}_{2}-\mathrm{CeCl}_{3}$ 沉淀明显增加, 伤害后 $1 \mathrm{~h}, \mathrm{H}_{2} \mathrm{O}_{2}$ 含量即 


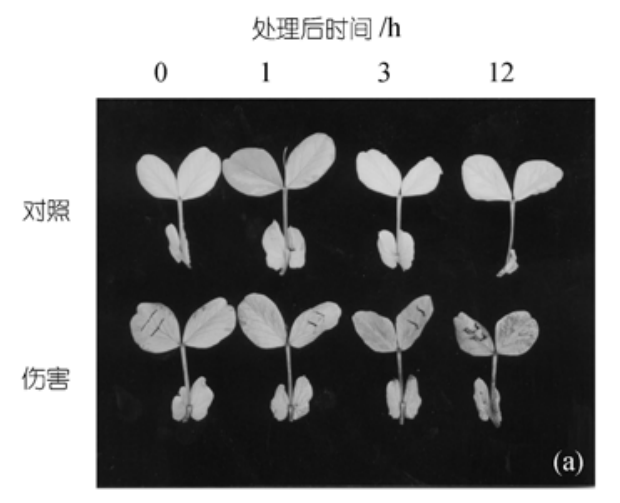

$1-3$

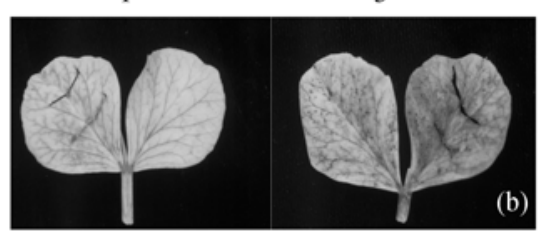

1

3

12

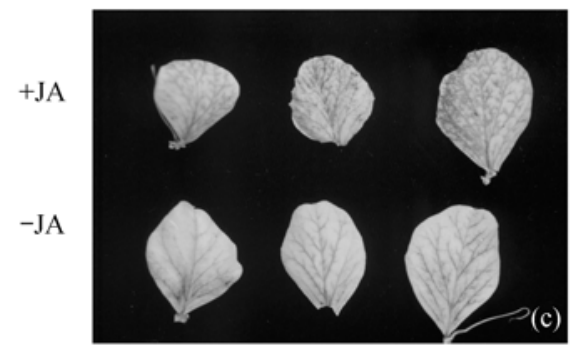

12

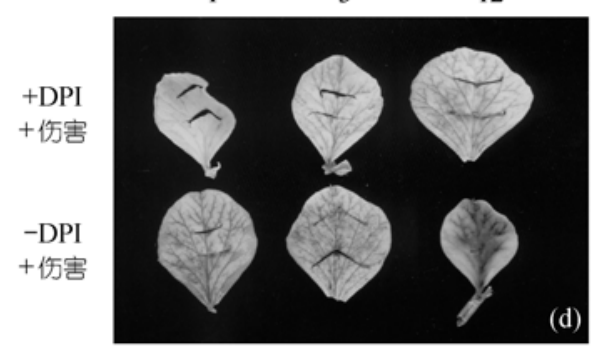

图 1 机械伤害和 JA 诱导踠豆叶片 $\mathrm{H}_{2} \mathrm{O}_{2}$ 产生的组织化学 定位

(a) 伤害处理后不同时间 $\mathrm{H}_{2} \mathrm{O}_{2}$ 的累积情况; (b) 伤害叶片及其邻近未伤 害叶片 $\mathrm{H}_{2} \mathrm{O}_{2}$ 的累积情况; (c) 外施 JA 后诱导 $\mathrm{H}_{2} \mathrm{O}_{2}$ 的累积情况; (d) 外施 DPI 抑制伤诱导 $\mathrm{H}_{2} \mathrm{O}_{2}$ 的累积情况

有所增加, 增加的沉淀颗粒主要位于质膜上, 在细胞 间隙也有少量分布(图 2(d)); 伤害后 $3 \mathrm{~h}, \mathrm{H}_{2} \mathrm{O}_{2}$ 含量急 剧增加，在质膜、细胞壁以及细胞间隙有大量密集的 沉淀颗粒分布(图 2(e)); 之后 $\mathrm{H}_{2} \mathrm{O}_{2}$ 含量逐渐减少, 到
伤害后 $12 \mathrm{~h}, \mathrm{H}_{2} \mathrm{O}_{2}$ 含量基本恢复至对照水平(图 2(f)). 外施 JA 处理后, 踠豆叶片叶肉细胞中 $\mathrm{H}_{2} \mathrm{O}_{2}$ 含 量、分布的动态变化均与伤害处理结果相似. JA 处理 后 $1 \mathrm{~h}$, 质膜上 $\mathrm{H}_{2} \mathrm{O}_{2}-\mathrm{CeCl}_{3}$ 沉淀颗粒即有所增加 (图 2(g)), JA 处理后 $3 \mathrm{~h}, \mathrm{H}_{2} \mathrm{O}_{2}$ 含量达到最大, 主要分布在 质膜、细胞壁和细胞间隙(图 2(h)), JA 处理后 $12 \mathrm{~h}$, $\mathrm{H}_{2} \mathrm{O}_{2}$ 含量减少，仅在质膜上有少量分布(图 2(i)).

我们还观察了伤害叶片相邻未伤害叶片、低节位 未伤害叶片叶肉细胞中 $\mathrm{H}_{2} \mathrm{O}_{2}$ 含量及其亚细胞分布, 结果显示, 伤害处理同样可以诱导上述未伤害叶片 $\mathrm{H}_{2} \mathrm{O}_{2}$ 含量增加(图 2(j), (k)). 其含量和分布的动态变 化与伤害处理结果相似(结果未全列出).

外源施加 DPI 后 $4 \mathrm{~h}$ ，再进行伤害和 JA 处理，叶 肉细胞中 $\mathrm{H}_{2} \mathrm{O}_{2}$ 含量较之未经 DPI 处理的明显减少(图 2(l), (m)).

\section{3 机械伤害对㛺亘叶片活性氧与抗氧化酶类活 性的影响}

我们进一步利用理化检测手段研究了活性氧和 抗氧化酶类的动态变化. 如图 3 所示, 无论是伤害叶 片还是未伤害邻近叶片和低节位远端叶片，伤害处 理后 $1 \mathrm{~h}, \mathrm{O}_{2}^{-*}$ 和 $\mathrm{H}_{2} \mathrm{O}_{2}$ 含量就明显增加, 伤害后 3 5 h, 其含量达到最大值, 随后迅速下降, 到伤害后 $24 \mathrm{~h}$, 与对照相比无显著差异. 且伤害叶片和远端未伤害 叶片活性氧峰值出现时间表现出同步性的特点, 仅 强度弱些, 而邻近未伤害叶片与伤害叶片相比, 活性 氧峰值出现时间稍有滞后，但强度相当(图 3(a), (b)).

伤害处理后, 无论是伤害叶片还是未伤害叶片, 抗氧化酶类 SOD, APX, CAT 活性均迅速增强. SOD 和 APX 活性最大值出现在伤害后 3 5 h, 之后活性逐渐 下降, 到处理后期基本恢复至对照水平(图 3(c), (d)). 伤害处理后, CAT 活性迅速提高, 处理后 $5 \mathrm{~h}$ 活性达到 最大值, 之后有所下降, 但仍高于对照(图 3(e)).

\section{4 机械伤害后踠豆叶片 JA 胶体金免疫电子显微 镜定位}

本试验中，JA 的免疫胶体金电子显微镜定位结 果显示, JA 在踠豆叶片叶肉细胞中广泛存在, 主要分 布于细胞壁、叶绿体和细胞质中(图 4(a)). 伤害处理 后, 伤害叶片叶肉细胞中代表 JA 的金颗粒数量迅速 

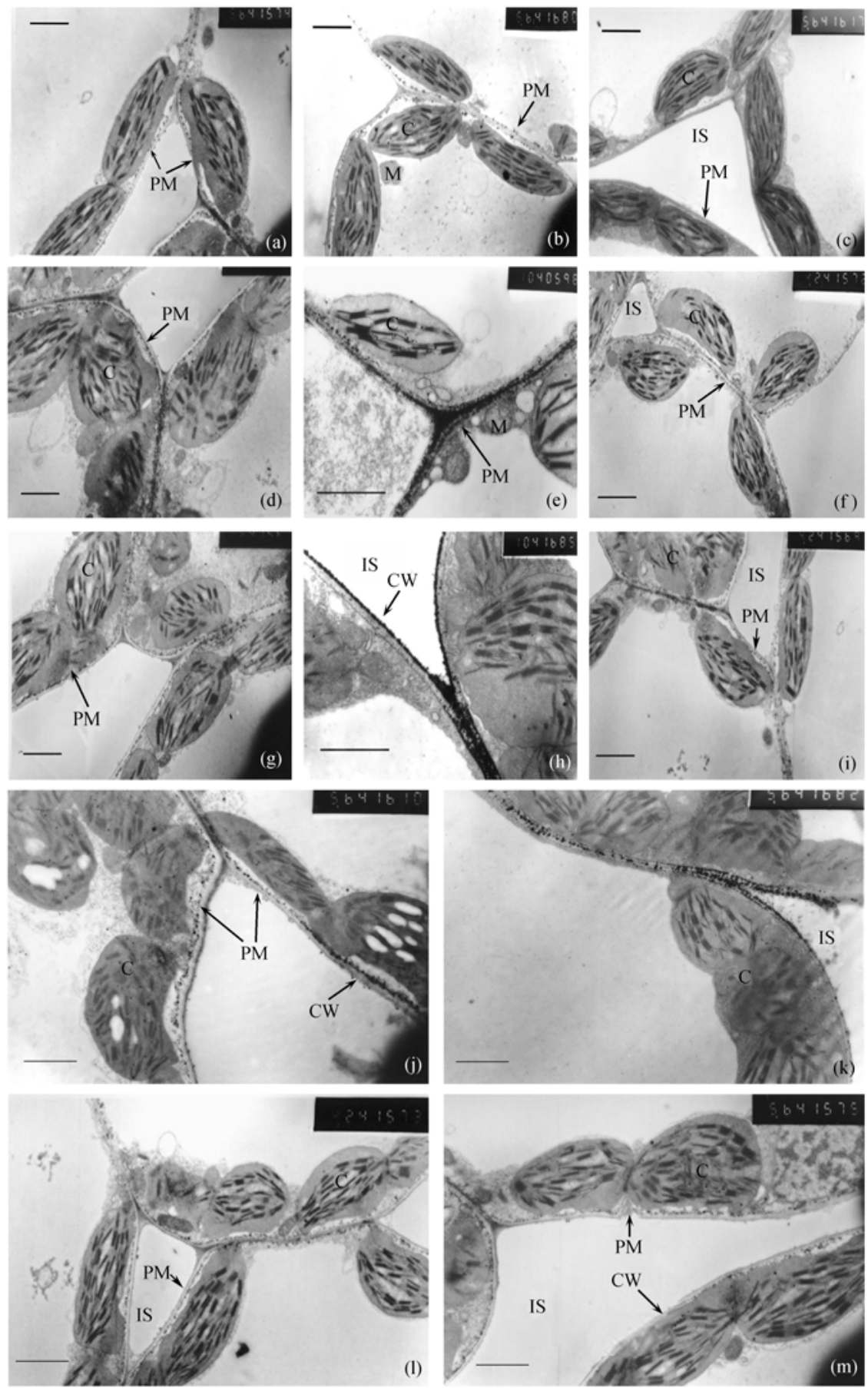

图 2 机械伤害和 JA 诱导踠豆叶片叶肉细胞产生 $\mathrm{H}_{2} \mathrm{O}_{2}$ 的细胞化学定位

(a)和(b) 未伤害的对照植株叶肉细胞质膜和叶绿体上有少量 $\mathrm{H}_{2} \mathrm{O}_{2}$ 分布. (c) 底物中不加 $\mathrm{CeCl}_{3}$ 的空白对照无沉淀显示. (d) 伤处理后 $1 \mathrm{~h}$, 伤害叶片 叶肉细胞中 $\mathrm{H}_{2} \mathrm{O}_{2}$ 累积量增加. (e) 伤处理后 $3 \mathrm{~h}, \mathrm{H}_{2} \mathrm{O}_{2}$ 在伤害叶片叶肉细胞的质膜、细胞壁、细胞间隙大量累积. (f) 伤处理后 $12 \mathrm{~h}$, 伤害叶片叶肉 细胞中 $\mathrm{H}_{2} \mathrm{O}_{2}$ 的累积量减少. (g) JA 处理后 $1 \mathrm{~h}, \mathrm{H}_{2} \mathrm{O}_{2}$ 在叶片叶肉细胞中的累积量增加. (h) JA 处理后 $3 \mathrm{~h}, \mathrm{H}_{2} \mathrm{O}_{2}$ 在叶片叶肉细胞中大量累积. (i) JA 处 理后 $12 \mathrm{~h}, \mathrm{H}_{2} \mathrm{O}_{2}$ 在叶片叶肉细胞中的累积量减少. (j) 伤处理后 $3 \mathrm{~h}, \mathrm{H}_{2} \mathrm{O}_{2}$ 在邻近未伤害叶片叶肉细胞中大量累积. (k) 伤处理后 $3 \mathrm{~h}, \mathrm{H}_{2} \mathrm{O}_{2}$ 在低节位 远端未伤害叶片叶肉细胞中大量累积. (l) DPI 处理后明显抑制伤诱导的 $\mathrm{H}_{2} \mathrm{O}_{2}$ 累积. (m) DPI 处理后明显抑制 JA 诱导的 $\mathrm{H}_{2} \mathrm{O}_{2}$ 累积. PM 示质膜; CW 示细胞壁; C 示叶绿体; $M$ 示线粒体; IS 示细胞间隙. 标尺示 $2 \mu \mathrm{m}$ 

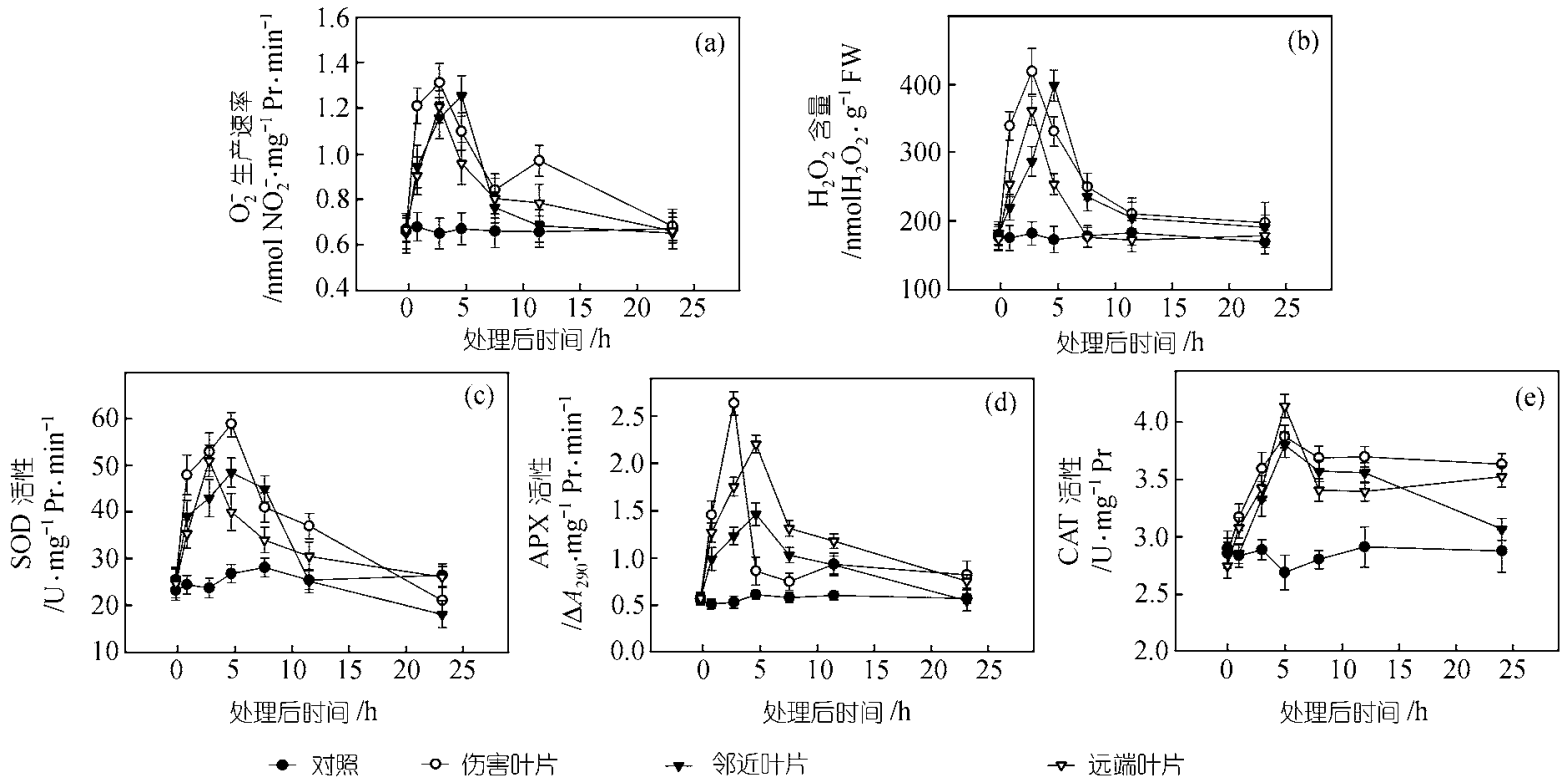

图 3 伤害处理后不同伤害叶片活性氧和抗氧化酶类活性的变化

(a) $\mathrm{O}_{2}^{-*}$ 产生速率; (b) $\mathrm{H}_{2} \mathrm{O}_{2}$ 含量; (c) SOD 活性; (d) APX 活性; (e) CAT 活性

增加, 增加的金颗粒主要集中分布在细胞壁上(图 4(b)); 在对照叶片中，维管束韧皮部篮管和伴胞分子 的细胞壁上也有金颗粒分布(图 4(c)), 伤害后该部位 金颗粒数量明显增加(图 4(d)). 对邻近和远端未伤害 叶片的 JA 定位结果显示, 伤害处理后，未伤害叶片 的叶肉细胞和维管束韧皮部篎管与伴胞分子中金颗 粒数量也明显增加, 且增加的数量主要位于细胞壁 上(图 4(e) (h)). 用免疫前的小鼠血清代替 JA 抗体的 对照和忽略一抗的对照中，基本上没有观察到金颗 粒标记(图 4(i) (j))).

\section{3 讨论}

植物生长、发育以及对生物和非生物胁迫反应的 过程中产生ROS, 大量的实验结果表明, $\mathrm{H}_{2} \mathrm{O}_{2}$ 可能作 为信号分子在防御反应中发挥作用 ${ }^{[4,5,10]}$. 但以往关 于 $\mathrm{H}_{2} \mathrm{O}_{2}$ 的研究多采用悬浮细胞系, 悬浮细胞系的缺 点是无法检测系统诱导作用. 以整株植株为材料的 研究表明, 外源激发子或病原菌只能诱导 $\mathrm{H}_{2} \mathrm{O}_{2}$ 的局 部迸发 ${ }^{[13,23]}$; 而Orozco-Cardenas等人 ${ }^{[4]}$ 和王源超等人 [24]的研究发现, $\mathrm{H}_{2} \mathrm{O}_{2}$ 可能被系统素或病原菌诱导系 统性产生, 并推测 $\mathrm{H}_{2} \mathrm{O}_{2}$ 可能通过植物的蒸腾作用运
输到全身, 从而诱导SAR建立. 我们在试验中同时利 用组织化学 $\mathrm{DAB}$ 染色和细胞化学 $\mathrm{CeCl}_{3}$ 沉淀以及理化 手段跟踪了伤害后 $\mathrm{H}_{2} \mathrm{O}_{2}$ 产生的时间、空间动态变化, 结果显示, 伤害可以诱导 $\mathrm{H}_{2} \mathrm{O}_{2}$ 在整株植株系统性产 生, 伤害处理后 $1 \mathrm{~h}$, 处理叶片就有 $\mathrm{H}_{2} \mathrm{O}_{2}$ 的累积, 3 5 $\mathrm{h}$ 后出现一个 $\mathrm{H}_{2} \mathrm{O}_{2}$ 迸发高峰, 随后逐渐下降; 同样, 在伤害叶片的邻近未伤害叶片以及低节位远端未伤 害叶片, $\mathrm{H}_{2} \mathrm{O}_{2}$ 变化趋势与伤害叶片相似, 也有一个迸 发高峰, 且与伤害叶片 $\mathrm{H}_{2} \mathrm{O}_{2}$ 迸发的时刻基本相同, 含 量基本相当, 由于采用伤害叶片的低节位叶片来观 察, 因此推测远端未伤害叶片中 $\mathrm{H}_{2} \mathrm{O}_{2}$ 迸发不可能通 过蒸腾作用从高节位伤害叶片输送而来，可能有其 他迅速传递的信号分子, 其传导的最终结果是诱导 植株系统迸发 $\mathrm{H}_{2} \mathrm{O}_{2}$.

我们利用 DAB 组织化学染色观察到, $\mathrm{H}_{2} \mathrm{O}_{2}$ 在维 管束组织和叶肉细胞中大量分布(图 1(a), (b)), 同时 利用 $\mathrm{CeCl}_{3}$ 沉淀法进一步观察了亚细胞水平上 $\mathrm{H}_{2} \mathrm{O}_{2}$ 分布和积累的动态变化, 结果显示, 在未伤害的对照 叶片, 质膜和叶绿体上有少量 $\mathrm{CeCl}_{3}$ 沉淀(图 2(a), (b)), 在液泡和其他细胞器如线粒体、细胞核上没有看到 $\mathrm{CeCl}_{3}$ 沉淀. Orozco-Cardenas 等人 ${ }^{[4]}$ 用百草枯处理材 


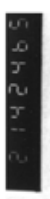

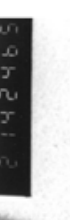

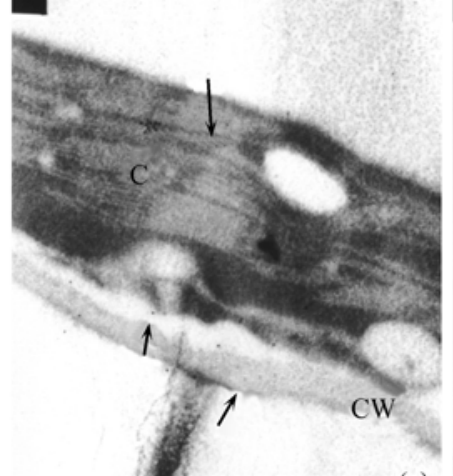

(a)
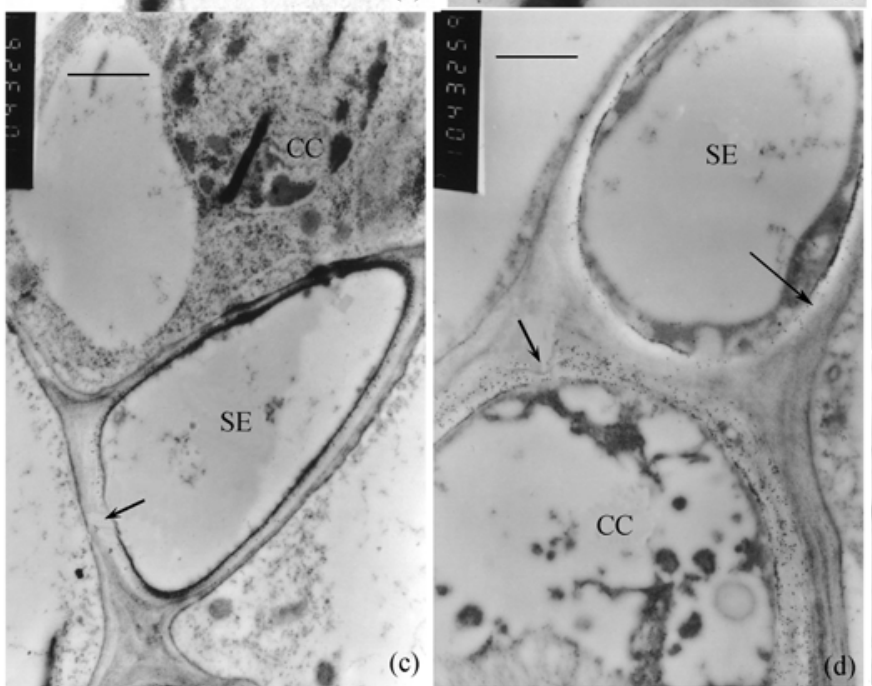

(b)
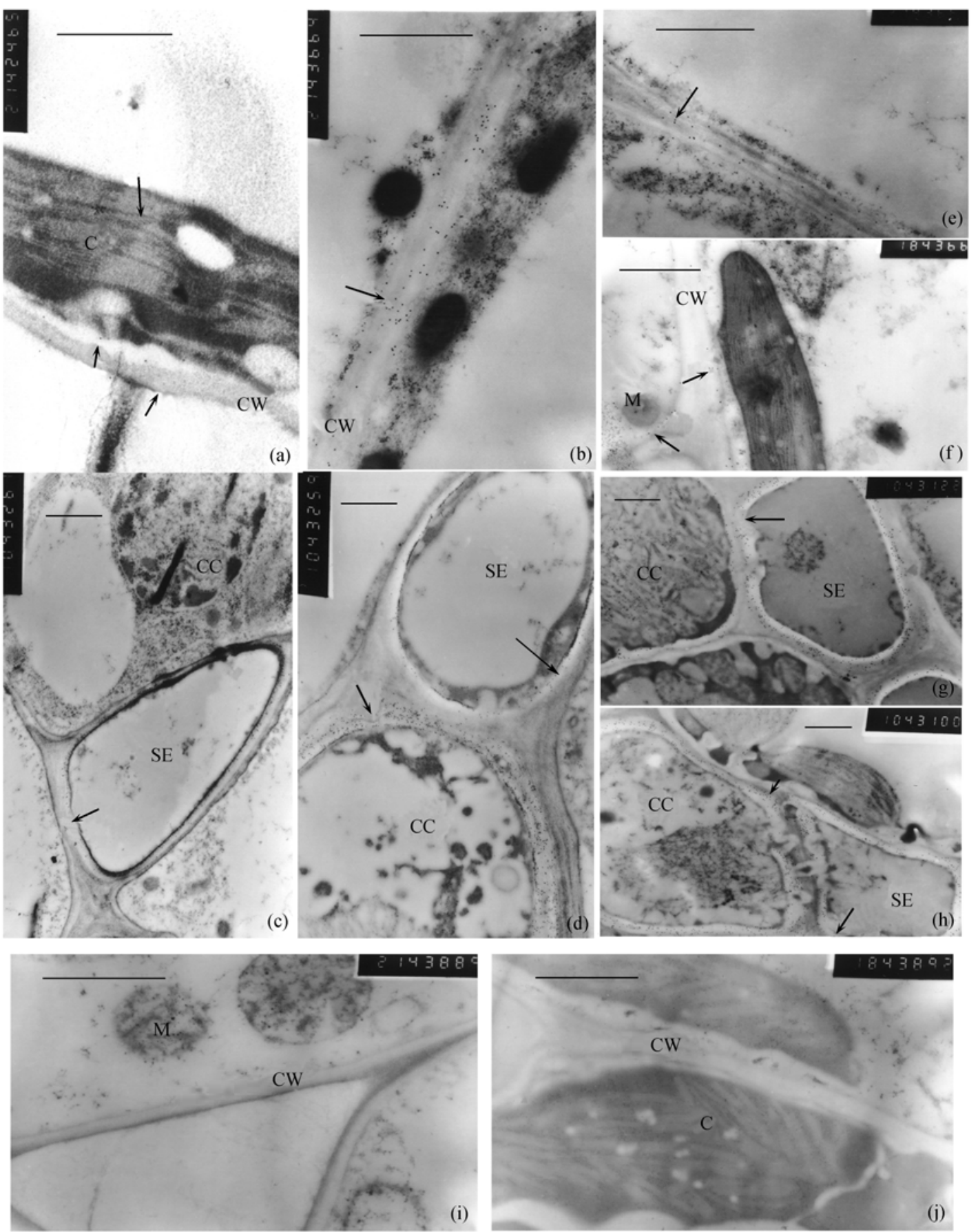

图 4 机械伤害后豌豆叶片 JA 胶体金免疫电子显微镜定位

(a) 未伤害的对照植株叶片叶肉细胞细胞壁、叶绿体和细胞质中有少量金颗粒分布. (b) 伤处理后 $3 \mathrm{~h}$, 伤害叶片叶肉细胞中金颗粒数量明显增加. (c) 未伤害的对照植株叶片韧皮部笁管和伴胞分子中有金颗粒分布. (d) 伤处理后 $3 \mathrm{~h}$, 伤害叶片韧皮部笁管和伴胞分子中金颗粒数量明显增加. (e) 伤处理后 $3 \mathrm{~h}$, 邻近未伤害叶片叶肉细胞中金颗粒数量明显增加. (f) 伤处理后 $3 \mathrm{~h}$, 远端未伤害叶片叶肉细胞中金颗粒数量明显增加. (g) 伤处理后 $3 \mathrm{~h}$, 邻近未伤害叶片韧皮部篮管和伴胞分子中金颗粒数量明显增加. (h) 伤处理后 $3 \mathrm{~h}$, 远端未伤害叶片韧皮部篎管和伴胞分子中金颗粒数量明显 增加. (i) 用免疫前鼠血清代替 JA 抗体的对照基本上没有金颗粒标记. (j) 不加 JA 抗体的对照也观察不到金颗粒标记. CW 示细胞壁; C 示叶绿体;

M 示线粒体; SE 示笁管; CC 示伴胞. 标尺示 $1 \mu \mathrm{m}$ 
料, 在叶绿体上发现有 $\mathrm{CeCl}_{3}$ 沉淀, 说明 $\mathrm{CeCl}_{3}$ 能够穿 透质膜进入细胞内部, 本试验结果表明, 在正常生长 的植株叶肉细胞中, 质膜和叶绿体可能是形成 $\mathrm{H}_{2} \mathrm{O}_{2}$ 的主要部位. 伤害处理后, 叶片叶肉细胞中 $\mathrm{H}_{2} \mathrm{O}_{2}$ 含量 较对照明显增加, 伤后 $1 \mathrm{~h} \mathrm{H}_{2} \mathrm{O}_{2}$ 即有所增加, 到伤害 处理后 $3 \mathrm{~h}, \mathrm{H}_{2} \mathrm{O}_{2}$ 大量产生, 并密集分布于质膜、细胞 壁和细胞间隙, 而叶绿体中 $\mathrm{H}_{2} \mathrm{O}_{2}$ 含量无明显变化(图 2(d) (f)), 推测质膜和细胞壁可能是伤害诱导 $\mathrm{H}_{2} \mathrm{O}_{2}$ 产 生的主要部位. 目前关于氧化突发活性氧的来源争 论点很多，根据现有的报道，主要有两种模式，质膜 $\mathrm{NADPH}$ 氧化酶 ${ }^{[25,26]}$ 和细胞壁 $\mathrm{pH}$ 依赖的过氧化物酶 ${ }^{[27]}$ 作用模式. 本试验用 NADPH氧化酶专一性抑制剂 DPI来检测对伤害诱导 $\mathrm{H}_{2} \mathrm{O}_{2}$ 生成的影响, 结果表明, DPI对伤害诱导 $\mathrm{H}_{2} \mathrm{O}_{2}$ 形成具有明显抑制作用(图 1(d), 图 2(l) (m)), 而 $\mathrm{H}_{2} \mathrm{O}_{2}$ 亚细胞观察结果也显示, 其主要 分布于质膜和细胞壁上, 这与质膜 $\mathrm{NADPH}$ 氧化酶存 在部位以及它催化 $\mathrm{H}_{2} \mathrm{O}_{2}$ 形成的原理相一致, 因此, 我 们推测 $\mathrm{NADPH}$ 氧化酶可能是氧化突发 $\mathrm{H}_{2} \mathrm{O}_{2}$ 形成的关 键酶.

活性氧在植物健康细胞的正常代谢中可以形成, 但如果积累的量过高就会产生细胞毒性. 本试验观 察到, 即使氧化迸发阶段 $\mathrm{H}_{2} \mathrm{O}_{2}$ 大量产生, 植物叶片组 织细胞结构仍很完整，没有引起细胞崩溃和死亡(图 2(e)，(h)). 研究结果表明，植物在长期进化过程中自 身形成多种活性氧清除机制，其中SOD, CAT, APX等 就是重要的活性氧清除酶类，它们协同作用可以将 $\mathrm{O}_{2}^{--}$和 $\mathrm{H}_{2} \mathrm{O}_{2}$ 转变为稳定的 $\mathrm{H}_{2} \mathrm{O}$ 和 $\mathrm{O}_{2}$, 从而保护膜脂免 遭过氧化损伤 [28]. 本试验中, 伤害处理后, 3 种抗氧 化酶活性均呈上升趋势(图 3(c) (e)), 可能在清除活 性氧过度累积中发挥重要作用, 而活性氧与抗氧化 酶类的协调作用可能是有效诱导伤害防御反应产生 的重要环节。

$\mathrm{JA}$ 是近年来发现的一种新型植物激素，大量的 研究结果表明, JA与植物抗性密切相关 ${ }^{[2 \sim 4]}$, 特别是 JA作为伤信号分子的作用越来越引起人们的关注 ${ }^{[1]}$. 但关于伤害防御反应中, JA与活性氧的关系还了解不 多. 我们研究了外施 $\mathrm{JA}$ 对 $\mathrm{H}_{2} \mathrm{O}_{2}$ 的影响, 结果显示, JA 同伤害处理一样也可以诱导 $\mathrm{H}_{2} \mathrm{O}_{2}$ 的产生, 并在处理 后 3 h有一个迸发高峰(图 1(c), 图 2(g) (i)), 这暗示
$\mathrm{H}_{2} \mathrm{O}_{2}$ 可能在JA下游发挥作用. 利用放射示踪技术, 在蚕豆 ${ }^{[29]}$ 中研究证实, $\mathrm{JA}$ 可以作为可移动的分子在 整株传递; Baldwin等人 ${ }^{[30]}$ 观察到，烟草叶片受伤后 伤害部位和未受伤的根系内源JA水平迅速增加. 我 们利用JA胶体金免疫电子显微镜定位技术，观察了 伤害处理后JA在叶肉细胞和维管组织中的分布与数 量变化, 结果显示, 伤害处理后, 金颗粒不仅在伤害 叶片，而且在未伤害叶片均明显增加，且增加的金颗 粒主要位于叶肉细胞的细胞壁和韧皮部篎管与伴胞 分子的细胞壁上(图 4). 这暗示着,一方面，JA对伤害 能做出快速应答，含量迅速增加; 另一方面, JA可能 从伤害位点通过质外空间如叶肉细胞细胞壁和维管 束韧皮部篎管与伴胞分子运输, 将伤害信息传递到 整株，进而诱导下游反应，如 $\mathrm{H}_{2} \mathrm{O}_{2}$ 系统性迸发等. 然 而，植物细胞是如何接受JA信息的？接受到JA信息 后又如何激活 $\mathrm{H}_{2} \mathrm{O}_{2}$ 产生，其作用的机制是怎样的? 这些都是值得进一步深入研究的问题.

致谢 感谢南京农业大学周嚄教授和甘立军博士馈 赠抗 JA 单克隆抗体.

\section{参考文献}

1 León J, Rojo E, Sanchez-Serrano J J. Wound sigmalling in plants. J Exp Bot, 2001, 354(52): 1 9[DOI]

2 Cohen Y, Gisi U, Niderman T. Local and systemic protection against Phytophthora infestans induced in potato and tomato plants by jasmonic acid and jasmonic methyl ester. Phytopanthology, 1993, 83: 1054 1062

3 Baldwin I T, Zhang Z P, Diab N. Quantification, correlations and manipulations of wound-induced changes in jasmonic acid and nicotine in Nicotiana sylvestris. Planta, 1997, 201: 397 404[DOI]

4 Orozco-Cardenas M, Narvaez-Vasquez J, Ryan C A. Hydrogen peroxide acts as a second messenger for the induction of defense genes in tomato plants in response to wounding, systemin, and methyl jasmonate. The Plant Cell, 2001, 13: 179 191[DOI]

5 Neuenschwander U, Vernooij B, Friedrich L, et al. Is hydrogen peroxide a second messenger of salicylic acid in systemic acquired resistance? Plant J, 1995, 8: 227 233[DOI]

6 Hammond-Kosack K E, Jones J D G. Resistance gene-dependent plant defense responses. The Plant Cell, 1996, 8: 1773 1791[DOI]

7 Doke N. Involvement of superoxide anion generation in the hypersensitive response of potato tuber tissue to infection with an incompatible race of Phytophthora infestans and to the hyphal wall components. Physiol Plant Path, 1983, 23: 345 357

8 Yahraus T, Chandra S, Legendre L, et al.. Evidence for a me- 
chanically induced oxidative burst. Plant Physiol, 1995, 109: 1259 1266

9 Amott T, Murphy T M. A comparison of the effects of a fungal elicitor and ultraviolet radiation on ion transport and hydrogen peroxide synthesis by rose cell. Environ Exp Bot, 1991, 31: 209 216[DOI]

10 Orozco-Cardenas M L, Ryan C A. Hydrogen peroxide is generated systemically in plant leaves pathway. Proc Natl Acad Sci USA, 1999, 96: 6553 6557[DOI]

11 Thordal-Christensen $\mathrm{H}$, Zhang Z, Wei Y, et al. Subcellular localization of $\mathrm{H}_{2} \mathrm{O}_{2}$ in plants: $\mathrm{H}_{2} \mathrm{O}_{2}$ accumulation in papillae and hypersensitive response during the barley-powdery mildew interaction. Plant J, 1997, 11: 1187 1194[DOI]

12 Orozco-Cardenas M, Ryan C A. Hydrogen peroxide is generated systemically in plant leaves by wounding and systemin via the octadecanoid pathway. Proc Natl Acad Sci USA, 1999, 96: 6553 6557[DOI]

13 Bestwick C S, Brown I R, Bennett M H R, et al. Localization of hydrogen peroxide accumulation during the hypersensitive reaction of lettuce cells to Pseudomonas syringae pv phaseolicola. The Plant Cell, 1997, 9: 209 221[DOI]

14 Pellinen R, Palva T, Kangasjarvi J. Subcellular localization of ozone-induced hydrogen peroxide production in birch (Betula pendula) leaf cells. Plant J, 1999, 20: 349 356[DOI]

15 Patterson B D, Mackae E A, Ferguson I B. Estimation of hydrogen peroxide in plant extracts using titanium (IV). Anyl Biochem, 1984, 139: 487 492

16 Dhindsa R S, Dhindsa P P, Thrope T A. Leaf senescence; correlated with increased levels of membrane permeability and lipid peroxidation, and decreased levels of superoxide dismutase and catalase. J Exp Bot, 1984, 3: 93 101

17 EI-Moshaty F I B, Pike S M, Novachy A J. Lipid peroxidation and superoxide production in cowpea (Vigna unguiculata)leaves infection with tobacco ring spot virus of southern bean mosaic virus. J Physiol Mol Plant Pathol, 1993, 43: 109 119[DOI]

18 王爱国, 罗广华. 植物的超氧物自由基与羟胺反应的定量关系. 植物生理学通讯, 1990, 6: 55 57

19 苏新国, 郑永华, 冯 否, 等. 外源 Me-JA 对菜用大豆荚采后
衰老和腐烂的影响. 植物生理与分子生物学学报, 2003, 29(1): $52 \sim 58$

20 沈文弹, 徐朗莱, 叶茂丙, 等. 抗坏血酸过氧化物酶活性测定 的探讨. 植物生理学通讯, 1996, 32(3): 203 205

21 Zhang D P,Lu Y M, Wang Y Z, et al. Acid inverters is predominately localized to cell walls of both the practically symplastically isolated sieve element/companion cell complex and parenchyma cells in developping apple fruits. Plant Cell and Eviron, 2001, 24(7): 691 702[DOI]

22 王永章, 张大鹏. 发育过程中苹果果实的 $\beta$-淀粉酶: 活性、数量 变化和亚细胞定位. 中国科学, C 辑, 2002, 32(3): 202 210

23 Costet L, Cordilier S, Dorey S, et al. Relationship between localized acquired resistance(LAR) and the hypersensitive response(HR): HR is necessary for LAR to occur and salicylic acid is not sufficient to trigger LAR. Mol Plant-Microbe Interact, 1999, 8: 655 666

24 王源超, 张正光, 李 俊, 等. $\mathrm{H}_{2} \mathrm{O}_{2}$ 参与棉疫病菌 $90 \mathrm{kD}$ 蛋白激 发子诱导的烟草过敏反应和系统获得抗性. 植物生理与分子生 物学学报, 2003, 29(3): 185 191

25 Auh C K, Murphy T M. Plasma membrane redox enzyme is involved in the synthesis of $\mathrm{O}_{2}^{-*}$ and $\mathrm{H}_{2} \mathrm{O}_{2}$ by Phytophthora elicitor-stimulated rose cells. Plant Physiol, 1995, 107: 1241 1247

26 Xing X, Higgins V J, Blumwaid E. Race-specific elicitors of Cladosporium fulvum promote translation of cytosolic components of NADPH oxidase to the plasma membrane of tomato cells. The Plant Cell, 1997, 9: 249 259[DOI]

27 Peng M, Kuc J. peroxidase-generated hydrogen peroxide as a source of antifungal activity in vitro and on tobacco leaf disks. Phytopathol, 1992, 82: 696 699

28 Levine A. Oxidative stress as a regulator of environmental responses in plants. In: Lerner H R. Plant Responses to Environmental Stresses. New York: Marcel Dekker Inc, 1995, 247 264

29 Liu X, Zhang S Q, Lou C H, et al. Effect of localized scorch on the transport and distribution of exogenous jasmonic acid in Vicia faba. Acta Botanica Sinica, 2002, 44(2): 164 167

30 Baldwin I T, Zhang Z P, Diab N, et al. Quantification, correlation and manipulations of wound-induced changes in jasmonic acid and nicotine in Nicotiana sylvetris. Planta, 1997, 201: 397 404[DOI] 